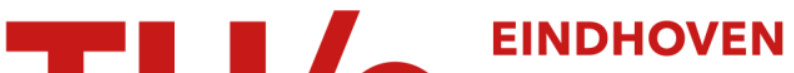 UNIVERSITY OF TECHNOLOGY
}

\section{Be delta-doped layers in GaAs imaged with atomic resolution using scanning tunneling microscopy}

Citation for published version (APA):

Johnson, M. B., Koenraad, P. M., van der Vleuten, W. C., Salemink, H. W. M., \& Wolter, J. H. (1995). Be deltadoped layers in GaAs imaged with atomic resolution using scanning tunneling microscopy. Physical Review

Letters, 75(8), 1606-1609. https://doi.org/10.1103/PhysRevLett.75.1606

DOI:

10.1103/PhysRevLett.75.1606

Document status and date:

Published: 01/01/1995

Document Version:

Publisher's PDF, also known as Version of Record (includes final page, issue and volume numbers)

Please check the document version of this publication:

- A submitted manuscript is the version of the article upon submission and before peer-review. There can be important differences between the submitted version and the official published version of record. People interested in the research are advised to contact the author for the final version of the publication, or visit the $\mathrm{DOI}$ to the publisher's website.

- The final author version and the galley proof are versions of the publication after peer review.

- The final published version features the final layout of the paper including the volume, issue and page numbers.

Link to publication

\section{General rights}

Copyright and moral rights for the publications made accessible in the public portal are retained by the authors and/or other copyright owners and it is a condition of accessing publications that users recognise and abide by the legal requirements associated with these rights.

- Users may download and print one copy of any publication from the public portal for the purpose of private study or research.

- You may not further distribute the material or use it for any profit-making activity or commercial gain

- You may freely distribute the URL identifying the publication in the public portal.

If the publication is distributed under the terms of Article 25fa of the Dutch Copyright Act, indicated by the "Taverne" license above, please follow below link for the End User Agreement:

www.tue.nl/taverne

Take down policy

If you believe that this document breaches copyright please contact us at:

openaccess@tue.nl

providing details and we will investigate your claim. 


\title{
Be Delta-Doped Layers in GaAs Imaged with Atomic Resolution Using Scanning Tunneling Microscopy
}

\author{
M. B. Johnson, ${ }^{1, *}$ P. M. Koenraad, ${ }^{2}$ W. C. van der Vleuten, ${ }^{2}$ H. W. M. Salemink, ${ }^{1}$ and J. H. Wolter ${ }^{2}$ \\ ${ }^{1}$ IBM Research Division, Zurich Research Laboratory, 8803 Rüschlikon, Switzerland \\ ${ }^{2}$ Physics Department, Eindhoven University of Technology, 5600 MB Eindhoven, The Netherlands
}

(Received 21 December 1994)

\begin{abstract}
We present first results of the analysis of molecular beam epitaxy-grown Be:GaAs delta-doped layers with atomic resolution. These were obtained using cross-sectional scanning tunneling microscopy. At low Be areal density, the width of the delta layers is $1 \mathrm{~nm}$, whereas at higher Be areal densities the spread of the layers is appreciable and the widths are approximately $5 \mathrm{~nm}$. This spreading is mostly symmetric around the intended position of the delta-doped layer, and is ascribed to drift resulting from the Coulombic repulsion between the ionized dopants at growth temperature. Structure in the spatial distribution of dopants within the delta layer also reflects this repulsion.
\end{abstract}

PACS numbers: 73.20.Dx, 61.16.Ch, 61.72.Ss, 71.55.Gs

As the size of semiconductor structures decreases, the dopant distributions become narrower, and the dopant density has to increase. Ultimately one tries to confine dopants to a single atomic plane. Such planes of dopants, called delta-doped ( $\delta$-doped) layers $[1,2]$, are achieved using molecular beam epitaxy (MBE) and related techniques for various dopants in III-V and group IV material systems, most notably $\mathrm{Be}$ and $\mathrm{Si}$ in GaAs $[3,4]$. The transport properties of $\delta$ layers are currently being studied and modeled [5], including the effects of dopant ordering [6].

For a fundamental understanding of $\delta$ layers, it is important to understand their spatial characteristics on the atomic scale. Conventionally, $\delta$ layers are studied using capacitance-voltage $(C-V)$ analysis [1] (electrical sensitivity) or secondary ion mass spectroscopy (SIMS) [1] (chemical sensitivity). However, neither technique has sufficient spatial resolution to resolve atomic details. Recently, transmission electron microscopy [7] and x-ray diffractometry [8] have been used to study $\delta$ doped layers but, due to limited sensitivity, only highly doped layers ( 0.5 of a monolayer) can be studied. Crosssectional scanning tunneling microscopy (XSTM) is a technique that is sensitive to dopants with atomic or nearatomic resolution in all spatial dimensions. Individual, activated $\mathrm{Be}, p$-type dopants in epitaxial GaAs were observed [9,10], followed by $\mathrm{Si}[11]$ and $\mathrm{Zn} \mathrm{[12]} \mathrm{in} \mathrm{bulk}$ GaAs, as well as Be in modulation-doped structures [13]. This unprecedented spatial resolution allows XSTM to study $\delta$-doped layers down to the atomic scale, as we demonstrate here.

In this Letter, we present first results on Be:GaAs $\delta$ doped layers with atomic resolution using XSTM. At low areal density $\left(\leq 10^{13} \mathrm{~cm}^{-2}\right)$, a $\delta$-layer width of 1 $\mathrm{nm}$ is observed, in marked contrast to the appreciable spreading to approximately $5 \mathrm{~nm}$ at higher concentrations $\left(\geq 3 \times 10^{13} \mathrm{~cm}^{-2}\right.$ ). This spreading is symmetric around the intended position of the layer and is ascribed to the Coulombic repulsion between the ionized dopant atoms.
In this study, a single epitaxial structure consisting of three sets of a series of four $\delta$-doped layers was used. The epitaxial GaAs layers were grown on $p+\operatorname{GaAs}(001)$ with on-axis orientation $\left(0.0^{\circ} \pm 0.2^{\circ}\right)$ at a substrate temperature of $480^{\circ} \mathrm{C}$, and a GaAs growth rate of approximately 1 bilayer per second. The Be $\delta$-doped layers were deposited during growth interrupts lasting between 10 and $360 \mathrm{~s}$ to produce intended two-dimensional doping concentrations of $3 \times 10^{12}, 1 \times 10^{13}, 3 \times 10^{13}$, and $1 \times 10^{14}$ (labeled $\delta_{1}-\delta_{4}$, respectively). A 25-nm-thick layer of nominally undoped GaAs was grown between each $\delta$ layer. The entire structure consists of a buffer layer, followed by the three identically grown sets of four $\delta$-doped layers, each set separated by 2.5 -nm-thick, Be-doped $\mathrm{Al}_{0.2} \mathrm{Ga}_{0.8} \mathrm{As}$ marker layers, followed by a $p+$ cap layer. Polaron $C-V$ and SIMS analyses [14] were performed on these layers, and a SIMS analysis of the cap layer served to calibrate the XSTM-measured doping concentrations. XSTM measurements were performed using an ultrahigh vacuum (UHV) STM with a base pressure of $1 \times 10^{-11}$ mbar on samples cleaved in vacuo to yield atomically flat, electronically unpinned (110) surfaces cross sectional to the [001] growth direction $[9,10]$.

Figure 1(a) shows a large-scale XSTM As-related image across the complete second set of $\delta$-doped layers, $\delta_{1}$ through $\delta_{4}$, as well as the marker layers $(M)$. Figure 1(b) shows an enlarged view of the smaller sections of $\delta_{1}$ and $\delta_{2}$ marked in Fig. 1(a). Figure 1(a) shows atomic corrugation in the [001] direction, whereas Fig. 1(b) shows atomic corrugation in both the [001] and [1 10$]$ directions. Several distinctive features can be observed as discussed in our previous work $[9,13]$; important for the present work are the white hillock features (the nearsurface dopants) situated in the proximity of the $\delta$-doped positions $\left(\delta_{1}-\delta_{4}\right)$.

The hillock features are due to the presence of nearsurface ionized Be dopants; note that no hillocks are observed in the undoped spacer layers. The hillocks reflect

(C) 1995 The American Physical Society 

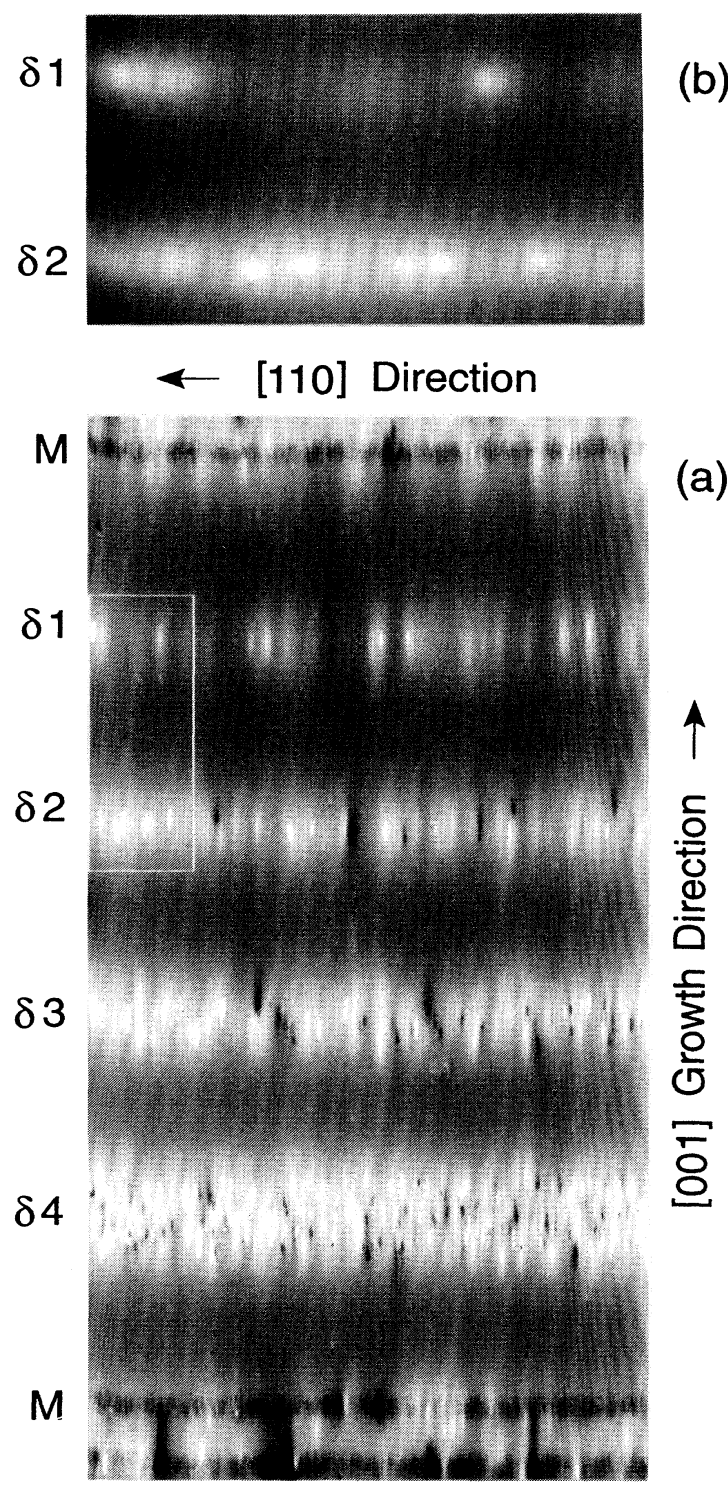

FIG. 1. STM images of Be $\delta$-doped layers. (a) Large-scale $\left(300 \times 120 \mathrm{~nm}^{2}\right)$ As-related image of the four $\delta$-doped layers of set 2 . The horizontal scale $(300 \mathrm{~nm})$ is strongly compressed. Tunneling conditions: sample bias $-1.0 \mathrm{~V}$ current $20 \mathrm{pA}$. The gray-scale range is $0.08 \mathrm{~nm}$ with a [001] corrugation of approximately $0.03 \mathrm{~nm}$. Electrically active Be dopants appear as white hillocks approximately $2.5 \mathrm{~nm}$ in diameter and up to $0.05 \mathrm{~nm}$ high. (b) Enlarged view $\left(54 \times 31 \mathrm{~nm}^{2}\right)$ of the section of the two lowest $\delta$-doped layers outlined in (a). Atomic corrugation in both directions is clearly observed, allowing dopants to be counted and their positions measured to the nearest bilayer in the plane shown.

an increase in the surface density of states near the valence band resulting from the scattering of carriers from the hydrogenlike ionized Be acceptor near the cleaved surface [10]. The radius of the hillock corresponds to the Bohr radius of the Be dopant $(2.5 \mathrm{~nm})$, and the height decreases the farther the acceptor site is located below the surface. Figures 1(a) and 1(b) allow the Be dopant position to be determined with atomic resolution in the [001] and $[1 \overline{1} 0]$ directions, and with near-atomic resolution in the $-[110]$ direction. Thus histograms of the positions of the dopants can be determined as shown in Fig. 2. Figures 2(a)-2(d) show the spread of the dopants in the [001] growth direction, and Figs. 2(e)-2(h) show the occurrence of hillock heights. Figures 2(a)-2(d) demonstrate that for the two lowest-concentration $\delta$ layers, the dopants spread only slightly out of a single plane, whereas for the two highest $\delta$ layers there is appreciable spreading. The zero position in the [001] histograms is the intended position of the $\delta$ layer and the position of the growth interrupt determined by counting the [001] GaAs atomic planes between the AlGaAs marker layers (estimated accuracy: 1 bilayer). Best-fit Gaussian distributions quantitatively assess the spreading $(\sigma)$ of the dopants out of the intended $\delta$-layer position, with a width of $w=2 \sigma$. Figures 2(e)-2(h) demonstrate that for the lowest concentration layers the depth distribution is near uniform from the lowest detectable hillock height (deepest dopants) to the maximum height (shallowest dopants) as expected for a random distribution [13]. For the highest concentration layers the distribution is narrower suggesting a loss in sensitivity to the deepest (low peak height) dopants, as discussed below. Analysis of XSTM images of the other two sets of $\delta$ layers reveals similar characteristics.

By comparing the XSTM-counted dopant density to the $C-V$ and SIMS-measured data, the electronic sensitivity of Be and the depth sensitivity of the XSTM technique can be investigated. Figure 3 shows the number of dopants measured as a function of the intended two-dimensional $\delta$-layer concentration. Data determined from SIMS and $C-V$ measurements as well as the expected line are plotted assuming a constant viewing depth of $1.5 \mathrm{~nm}$. This depth was determined by counting dopants in the STM images of the Be:GaAs cap layer, whose intended and SIMSmeasured doping concentration is $1 \times 10^{19} \mathrm{~cm}^{-3}$. The agreement between the three sets of XSTM-counted data is very good. The SIMS and $C-V$ data are in good agreement with the expected concentration for the lower concentrations. But there is a large difference between the XSTM-dopant counting data and the SIMS, the $C-V$, and the expected data. This is not due to a reduced activity of $\mathrm{Be}$ (the SIMS and $C-V$ agree well), rather it indicates that the depth sensitivity of the XSTM-dopant counting varies with dopant concentration, decreasing from approximately 2 to $0.5 \mathrm{~nm}$ for intended concentrations of $3 \times 10^{12}$ to $1 \times 10^{14} \mathrm{~cm}^{-2}$, respectively. We suspect that this is not due to the difficulty in resolving overlapping dopants, but to the reduction of the scattering cross section of the near-surface dopants at high density. The reduced cross section results from the increase in the excess energy of tunneling holes due to the change in band bending with doping density [13]. This variation of the depth sensitivity of dopant counting with XSTM indicates that 


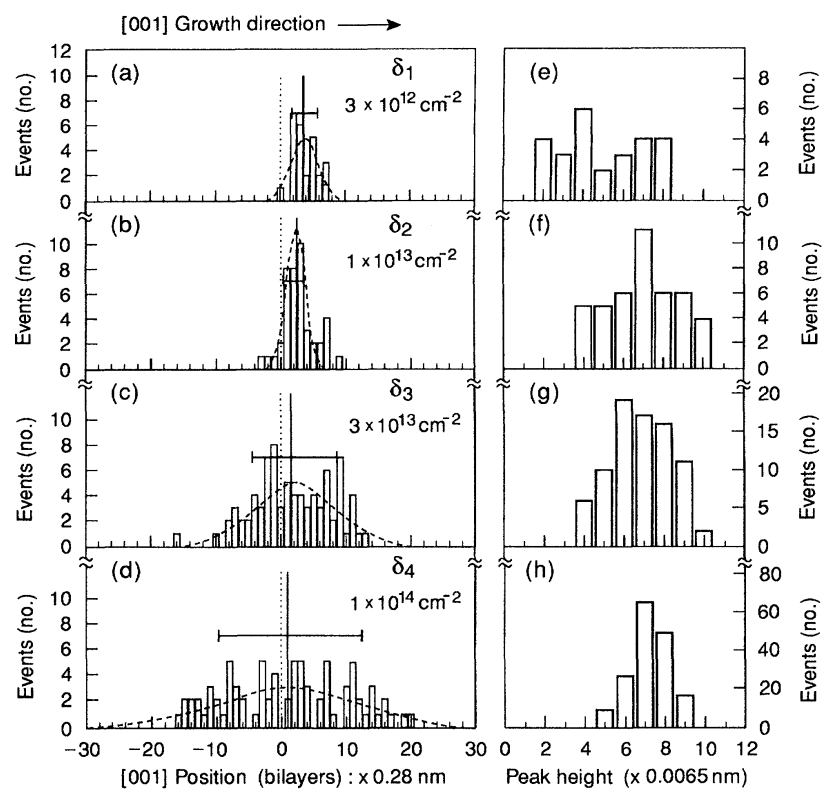

FIG. 2. Position-related histograms based on the STM images in Fig. 1(a). (a)-(d) Histograms of the [001] positions of the counted dopants for the $\delta$ layers in set 2, with growth direction indicated. A Gaussian fit (dotted) is used to determine the distribution's average position (solid) and width. The intended position of the $\delta$ layer is a dotted vertical line. (e)(h) Histograms of dopant height occurrence for the layers in set 2. Horizontal scale denotes apparent peak height in nanometers. Note that the relative height is reproducible within a given experiment, but might vary slightly on an absolute scale because of tip-to-tip variations.

careful analysis is necessary in order to determine dopant activity.

The [001] histograms in Fig. 2 clearly show that XSTM is an excellent technique to determine the spreading of $\delta$ layers: Ultimate bilayer resolution is achieved, many different layers can be studied independently as well as simultaneously, and the technique is sensitive to low areal dopant concentration. Both the lowest detectable concentration and the statistics are improved by larger scan areas. For the measurements described here $(300 \mathrm{~nm}$ in the [1]0] direction), a sensitivity to $\delta$ layers with $N_{2 \mathrm{D}}$ lower than approximately $5 \times 10^{11} \mathrm{~cm}^{-2}$ is achieved, and this would allow us to observe a $N_{3 \mathrm{D}}$ background of approximately $1 \times 10^{16} \mathrm{~cm}^{-3}$. The [001] histograms show a shift of 1 or 2 bilayers toward the surface. Such surface segregation is the result of the drift of the ionized $\mathrm{Be}$ in the presence of the near-surface $E$ field, which is built up by the pinned [001] growth surface [1]. This $E$ field is not responsible for the $\delta$-layer widening, because the observed $\delta$ layers remain symmetric. The situation is made clearer by plotting the $\delta$-layer width vs the intended doping concentration and vs time at growth temperature [Figs. 4(a) and 4(b)]. The $\delta$-layer width shows a striking dependence on concentration, which is explained by considering the drift due to the Coulomb repulsion of the ionized

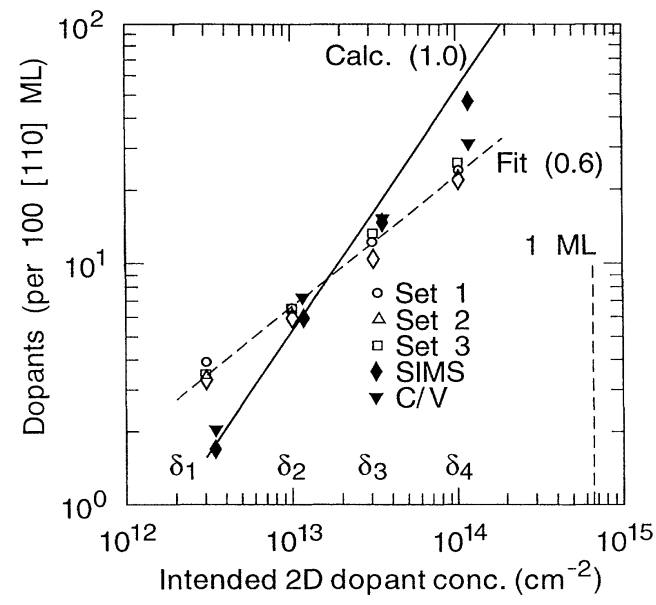

FIG. 3 Plot of measured vs intended doping concentration. Data obtained from counting the XSTM-imaged dopants are shown by open symbols, including the line of best fit (dotted, slope 0.6). Data from SIMS and $C-V$ are shown by solid symbols (offset), including the line calculated for a constant viewing depth sensitivity of $1.5 \mathrm{~nm}$ (solid, slope 1.0).

dopants within the $\delta$ layer that occurs at growth temperature [15]. The results of Monte Carlo modeling, which accounts for diffusion and drift, show that drift is less important for lower concentrations [16]. Furthermore, Monte Carlo modeling indicates that at higher concentrations a flat-topped [001] distribution is expected as observed in Fig. 2(d). Figure 4(b) confirms for the different sets of $\delta$ layers that the process responsible for the spreading takes place during bulk growth because the width is dependent on the time at growth temperature.

One of the most exciting prospects for the atomically resolved XSTM analysis of $\delta$-doped layers is the ability to measure the in-plane dopant distribution and thereby to look for in-plane structure and ordering at the atomic

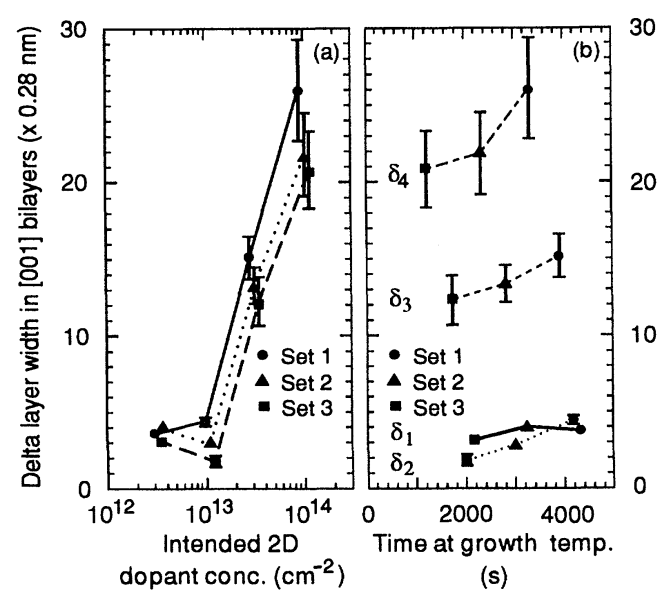

FIG. 4. Plot of layer width vs (a) intended concentration and (b) time at growth temperature. Data are shown for the three sets of four $\delta$ layers. 
level. Figure 5 shows the radial distribution function (frequency plot of dopant separation of all pairs of dopants) for $\delta_{1}, \delta_{2}$, and $\delta_{3}$ and the theoretical random curves. The measured data show that the occurrence of close-spaced neighbors is less probable, in sharp contrast to the flat distribution expected for a random distribution. The less probable occurrence of close neighbors is the consequence of the drift due to Coulombic repulsion between individual ionized dopants, which acts to move close-spaced dopants apart and results in an in-plane structure (short-range order) in the layers. The features in Fig. 5 at longer separations are statistical fluctuations, not oscillations, which would indicate long-range order as in liquids and solids. Thus only structure similar to that of a highly interacting gas is observed. Physically, this corresponds to dopants that strongly interact only when their Bohr diameters start to overlap.

The fact that the same Coulomb repulsion that acts to separate the dopants within the $\delta$ layer (in-plane) also acts to widen the layer (out-of-plane) indicates that long-range in-plane ordering is difficult to achieve. For this to take place, it may be important to exploit the anisotropy of the dopant mobility during the growth interrupt when the Be adatom is mobile on the growth surface.

In conclusion, we have demonstrated the use of XSTM to study $\delta$-doped layers in GaAs with atomic resolution. For Be:GaAs $\delta$-doped layers with low areal dopant density, the width of the doping plane is approximately $1 \mathrm{~nm}$ (4 bilayers), whereas at higher densities, apprecia-

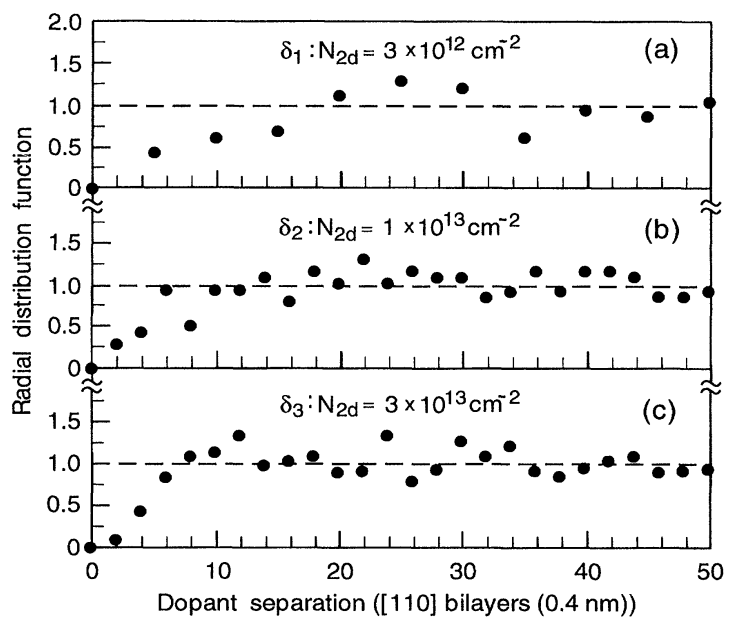

FIG. 5. Radial distribution function for $\delta_{1}, \delta_{2}$, and $\delta_{3}$ determined from the XSTM images (solid circles) and the theoretical random distribution (dotted line), showing the lower probability of $\mathrm{Be}: \mathrm{Be}$ close neighbors due to Coulombic repulsion. ble spreading to approximately $5 \mathrm{~nm}$ (20 bilayers) is observed. This spreading is mostly symmetric around the intended position of the layer and is ascribed to the Coulombic repulsion between the ionized dopants themselves. Structure in the spatial distribution of dopants within the $\delta$ layer is observed and also reflects this repulsion.

We acknowledge L. Perriard and H. Richard for sample preparation, F. Cardone for SIMS measurements, and B. Smalbrugge for $C-V$ measurements.

*Present address: Dept. of Physics and Astronomy, Univ. of Oklahoma, 440 West Brooks, Norman, OK 73072.

[1] See E.F. Schubert, Doping in III-V Semiconductors (Cambridge University Press, UK, 1993), and references therein.

[2] K. Ploog, J. Cryst. Growth 81, 304 (1987).

[3] J.J. Harris, J.B. Clegg, R. B. Beall, J. Castagne, K. Woodbridge, and C. Roberts, J. Cryst. Growth 111, 239 (1991).

[4] E. F. Schubert, J. M. Kuo, R. F. Kopf, H. S. Luftman, L. C. Hopkins, and N. J. Sauer, J. Appl. Phys. 67, 1969 (1990).

[5] E. F. Schubert, J. E. Cunningham, and W. T. Tsang, Solid State Commun. 63, 591 (1987).

[6] A. F. J. Levi, S. L. McCall, and P. M. Platzman, Appl. Phys. Lett. 54, 940 (1989).

[7] A. Ourmazd, J. Cunningham, W. Jan, J. A. Rentschler, and W. Schroeter, Appl. Phys. Lett. 56, 854 (1990).

[8] L. Hart, M. R. Fahy, R. C. Newman, and P.F. Fewster, Appl. Phys. Lett. 62, 2218 (1993).

[9] H. W. M. Salemink, M. B. Johnson, U. Maier, P. Koenraad, and O. Albrektsen, in Semiconductor Interfaces at the Sub-Nanometer Scale, edited by H.W. M. Salemink and M. D. Pashley, NATO ASI, Vol. 243 (Kluwer, Dordrecht, 1993).

[10] M. B. Johnson, O. Albrektsen, R. M. Feenstra, and H. W. M. Salemink, Appl. Phys. Lett. 63, 2923 (1993).

[11] J.F. Zheng, X. Liu, N. Newman, E. R. Weber, D. F. Ogletree, and M. Salmeron, Phys. Rev. Lett. 72, 1490 (1994).

[12] J. F. Zheng, M. B. Salmeron, and E. R. Weber, Appl. Phys. Lett. 64, 1836 (1994).

[13] M. B. Johnson, H. P. Meier, and H. W. M. Salemink, Appl. Phys. Lett. 63, 3636 (1993).

[14] See papers on $C-V$ and SIMS profiling in Proceedings of the 2nd International Workshop on Ultra-Shallow Doping Profiles, Raleigh, NC, March 1993 [J. Vac. Sci. Technol. B 12, 327ff (1994)]. Polaron $C-V$ is a liquid etch depth profiling the $C-V$ measurement technique.

[15] E.F. Schubert, G. M. Gilmer, R.F. Kopf, and H.S. Luftman, Phys. Rev. B 46, 15078 (1992).

[16] M. B. Johnson (to be published). 


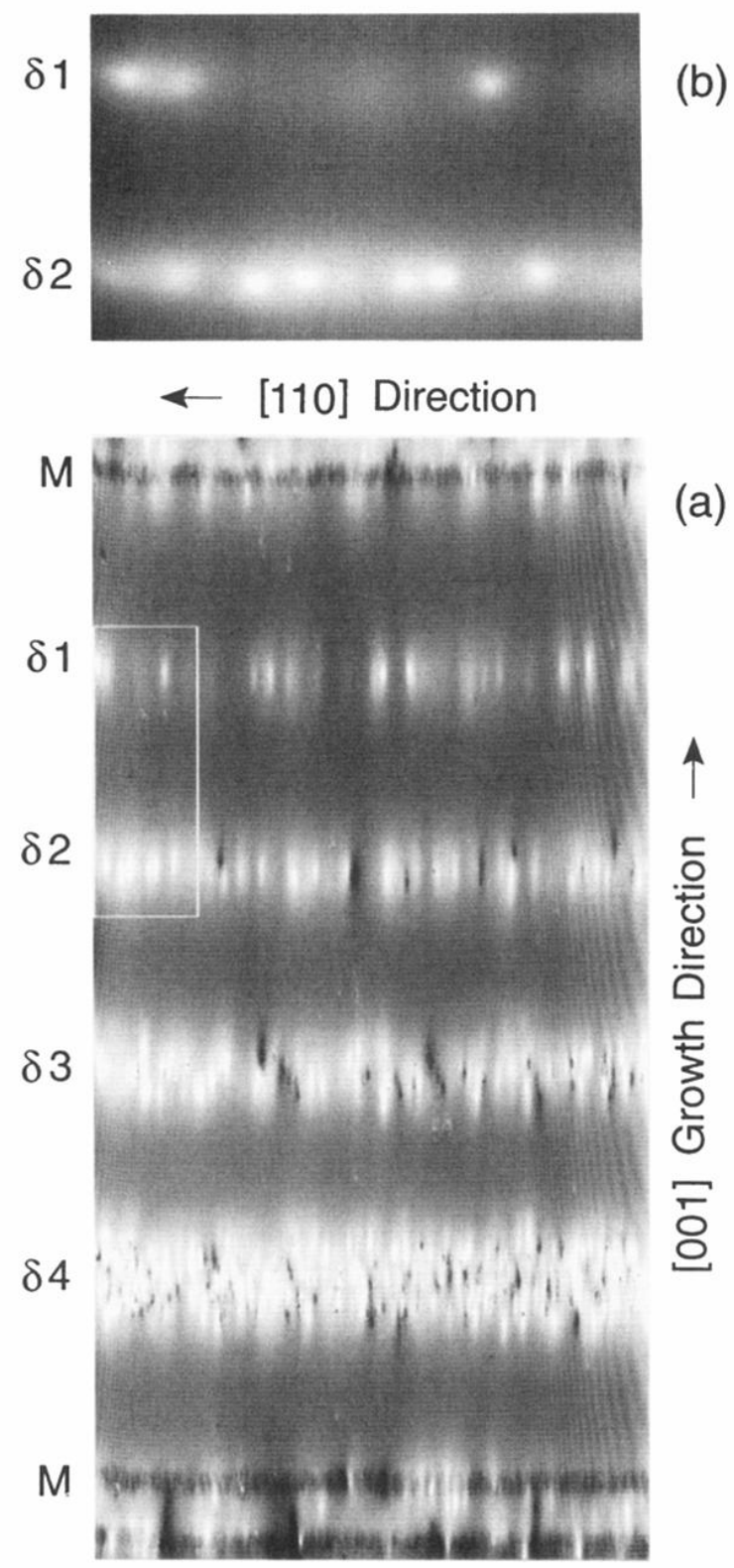

FIG. 1. STM images of Be $\delta$-doped layers. (a) Large-scale $\left(300 \times 120 \mathrm{~nm}^{2}\right)$ As-related image of the four $\delta$-doped layers of set 2 . The horizontal scale $(300 \mathrm{~nm})$ is strongly compressed. Tunneling conditions: sample bias $-1.0 \mathrm{~V}$ current $20 \mathrm{pA}$. The gray-scale range is $0.08 \mathrm{~nm}$ with a [001] corrugation of approximately $0.03 \mathrm{~nm}$. Electrically active Be dopants appear as white hillocks approximately $2.5 \mathrm{~nm}$ in diameter and up to $0.05 \mathrm{~nm}$ high. (b) Enlarged view $\left(54 \times 31 \mathrm{~nm}^{2}\right)$ of the section of the two lowest $\delta$-doped layers outlined in (a). Atomic corrugation in both directions is clearly observed, allowing dopants to be counted and their positions measured to the nearest bilayer in the plane shown. 